\title{
Terminal extinction performance of a running response following consistent and partial reinforcement and nonreinforcement
}

\author{
ROGER W. BLACK, JOSEPH SCHUMPERT \\ and \\ CHARLES WOODARD \\ University of South Carolina, Columbia, S.C. 29208
}

Rats were trained to traverse a straight runway under conditions of consistent reinforcement (CR), partial reinforcement (PR), or nonreinforcement (NR). Thereafter, all Ss received 2 extinction days of 40 nonreinforced trials each. The usual PRE was observed on Day 1 of extinction. During Day 2, PR and CR Ss ceased to respond differently and converged on the level of performance of the control (NR) group. Greater spontaneous recovery occurred for CR than PR Ss. These data suggest that the PRE disappears if the number of nonreinforced trials is sufficiently great to allow the extinction of incentive motivation.

One of the best known interpretations of the partial reinforcement effect (PRE) in instrumental reward conditioning is the frustration theory proposed by Amsel (e.g., 1958, 1967). According to this view, the acquisition of an instrumental response under appetitive reward involves the establishment, through classical conditioning, of an anticipatory reward mechanism (rg-sg), the motivational effects of which Spence (1956) labeled "incentive motivation," or K. Once rg-sg is sufficiently well established, the nonreinforcement of the instrumental response is assumed to result in frustration (Rf) and its associated cues (Sf). It is further assumed that an anticipatory frustration mechanism (rf-sf) becomes conditioned with repetitive nonreinforcement and that sf tends to elicit responses that interfere with the instrumental response. Thus, if a response which is acquired under consistent reinforcement (CR) is subjected to consistent nonreward (extinction), the situational cues which evoke the instrumental response will also come to elicit rf-sf, with an attendant decrement in the observed strength of the former. On the other hand, if the instrumental response is acquired under partial reinforcement (PR), the competing responses evoked by sf will tend to extinguish and sf will, in fact, become a portion of the stimulus complex which elicits the instrumental response. Extinction following PR will, therefore, result in much less interference with the instrumental response than that which occurs following $\mathrm{CR}$, i.e., the PRE is predicted.

Most studies of the PRE have been limited to a demonstration of greater response strength (e.g., faster running) on the part of PR Ss during the early trials of extinction or throughout a small number of extinction trials. Little attention has been given to "terminal extinction performance," i.e., performance following a large number of extinction trials. Thus, it is not clear whether the PRE is highly durable; nor is it clear whether frustration theory predicts great durability for the PRE.

The present study provided an opportunity to observe the performance of PR and CR Ss during the course of very extended extinction. Further, a control (NR) group was also employed which never received reward during "acquisition" or "extinction" and which thus provided a base level of performances in the absence of both frustration and incentive motivation. For this situation, several outcomes seem theoretically possible. For example, for CR Ss the intense frustration elicited in the goalbox on extinction trials may make that situation so aversive that Ss learn to avoid or "resist" entry to the goalbox. In this event, the goal speed of CR Ss may be depressed to a terminal extinction level below that of the NR control group. Further, since the instrumental response has been conditioned to sf for PR Ss, such Ss may continue to perform that response indefinitely and thus show terminal extinction performance at a level above that of either the CR or NR groups. Both of the preceding arguments lead to an expectation of highly persistent effects of acquisition reinforcement schedule on extinction performance. These arguments do not, however, take into account the fact that theoretically each nonreinforced extinction trial results not only in frustration but also in the extinction of incentive motivation (rg-sg). Thus, with a large number of extinction trials, incentive motivation for both CR and PR Ss should approach a "zero" or preacquisition value. Under this condition, frustration may also cease to occur. In this case, the late extinction performance of the CR and PR groups may converge to the level of performance exhibited by the NR Ss, for which both frustration and incentive motivation are also absent. The present experiment was designed to test these alternative interpretations of the frustration hypothesis.

\section{METHOD}

The Ss were 48 male albino rats about 90 days old at the beginning of training. All Ss were maintained on $10 \mathrm{~g}$ of lab chow per day for 14 days before and throughout the course of the experiment, with water being continuously available. The apparatus consisted of a $116.84-\mathrm{cm}$ straight runway, divided by horizontally sliding doors into $25.4-\mathrm{cm}$ goal- and startboxes. Photoelectronic circuitry provided measures of running time (recorded from the opening of the startbox until S reached a point $15.24 \mathrm{~cm}$ within the alley) and goal time (measured over 


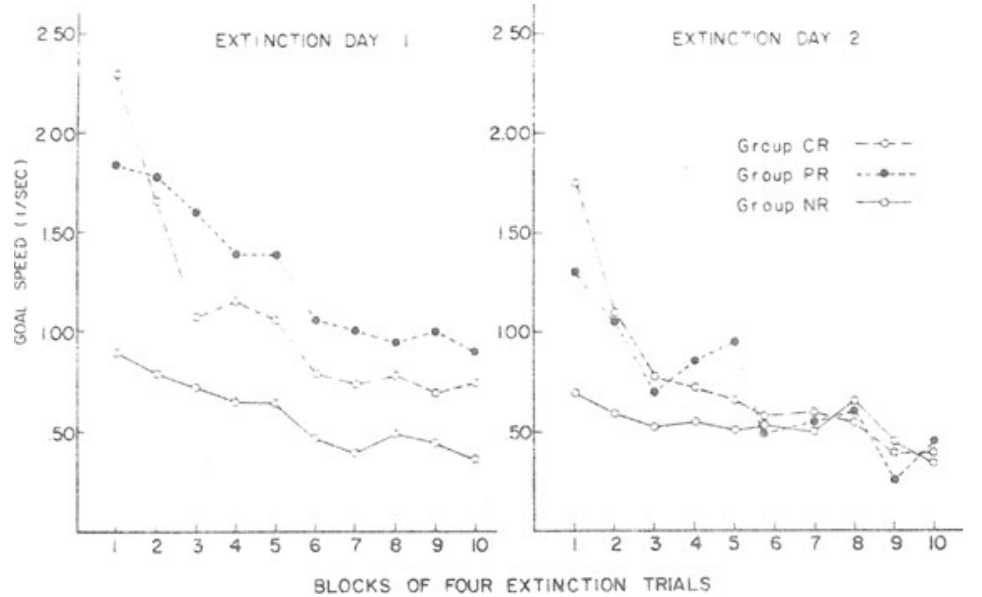

Fig. 1. Goal speeds for the three groups as a function of blocks of four extinction trials.

the final $15.24 \mathrm{~cm}$ of the alley to a point $15.24 \mathrm{~cm}$ within the goalbox).

A trial consisted of placing $S$ in the startbox and opening the startbox door. When $S$ entered the goalbox, the goalbox door was closed. The $S$ remained in the goalbox for $30 \mathrm{sec}$ or until it ate the available reward. Four acquisition trials per day were administered for 15 consecutive days. There were three independent groups of $16 \mathrm{Ss}$ each. For Group CR, each trial was reinforced with two $45-\mathrm{mg}$ Noyes pellets, while Group PR received two pellets on a random half of the trials, with no reward being present on the remaining trials. The Ss in Group NR received no reward on any trial. The Ss were run sequentially in squads of 12 , and the average intertrial interval was about 15 min during acquisition.

For all Ss, extinction began on the day following the final acquisition day and consisted of 40 nonreinforced trials per day on each of 2 consecutive days. On each trial $\mathrm{S}$ was confined in the unbaited goalbox for $30 \mathrm{sec}$, and the intertrial interval was about $20 \mathrm{~min}$.

\section{RESULTS AND DISCUSSION}

Before analysis, all individual running and goal times were converted to reciprocals $(1 / \mathrm{sec})$. Because the results for the two speed measures were essentially identical, only goal speeds are presented here. Figure 1 represents mean goal speed as a function of blocks of four extinction trials for both days of extinction. Analysis of performance on Day 1 indicated that goal speed for all groups reliably decreased $(F=31.59, \quad d f=9 / 405$, $\mathrm{p}<.001)$. During Day 1, Group PR had a higher goal speed than Group $\mathrm{CR}$, while both of these groups were superior to Group NR $(\mathrm{F}=11.88, \mathrm{df}=2 / 45, \mathrm{p}<.01)$. Similarly, the interaction of Groups by Trials was also significant $(\mathrm{F}=3.02, \mathrm{df}=18 / 405, \mathrm{p}<.05)$, reflecting the gradual convergence of the groups. Thus, the usual partial reinforcement effect occurred on Extinction Day 1.

Figure 1 also presents group mean goal speed on Extinction Day 2. Inspection of this figure suggests that the superiority of Group PR to Group CR had disappeared by this time. In addition, both of these groups appeared to be converging on the performance of Group NR, which had never been rewarded. Thus, the general decline in performance on Day 2 was statistically reliable $(F=18.13, d f=9 / 405, p<.001)$. Similarly, the convergence of the groups was also significant $(\mathrm{F}=2.32$, $\mathrm{df}=18 / 405, \mathrm{p}<.01)$. On the other hand, Group PR and Group CR did not differ from Group NR reliably on the last five blocks of extinction trials $(F=1.17, \mathrm{df}=2 / 45$, $\mathrm{p}>$.05).

The results of the present experiment clearly suggest that there are no "residual effects" of training under either PR or CR. Thus, neither was there a decrement in the performance of Group CR relative to Group NR nor did the initial superiority of Group PR (the PRE) persist indefinitely. Instead, the performance of both Groups $P R$ and $C R$ eventually became indistinguishable from that of Group NR, which never received reinforcement. This result supports the interpretation of Amsel's frustration theory presented earlier to the effect that repeated nonreward results in the extinction of rg-sg and, thus, in the disappearance of frustration. It should be noted that a large number of extinction trials was required to produce this effect. Thus, at the end of Extinction Day 1 (which itself provided a relatively large number of extinction trials), the PRE was still apparent and both Groups PR and CR were still superior to Group NR. It was not until the middle of Extinction Day 2, after more than 60 extinction trials, that Groups PR and CR became indistinguishable from Group NR. Nevertheless, performance beyond this point in extinction seemed to indicate that the effects of acquisition reinforcement schedule were completely "reversible."

A final interesting feature of the results in Fig. 1 is the spontaneous recovery apparent at the beginning of Extinction Day 2. Although both Groups PR and CR showed spontaneous recovery, the magnitude of the effect was substantially greater for the CR Ss. Thus, an analysis of performance of Groups CR and PR on the last trial block of Extinction Day 1 and the first block of Extinction Day 2 showed a significant increase in goal speed between days, i.e., a spontaneous recovery effect $(\mathrm{F}=8.44, \mathrm{df}=1 / 30, \mathrm{p}<.01)$. The greater magnitude of the spontaneous recovery effect for Group CR than for Group PR is confirmed by the significant Groups by Trial Blocks interaction $(F=5.30, d f=1 / 30, p<.05)$. 
Although it was clear that in the present experiment acquisition reinforcement schedule was a significant determinant of the amount of spontaneous recovery following extinction, the writers could find no previous report of this phenomenon nor any theoretical basis for expecting it. reward situations. Psychological Bulletin, 1958, 55, 102-119.

Amsel, A. Partial reinforcement effects on vigor and persistence: Advances in frustration theory derived from a variety of within-subjects experiments. In $K$. W. Spence and J. T. Spence (Eds.), The psychology of learning and motivation: Advances in research and theory. Vol. 1. New York: Academic Press, 1967.

Spence, K. W. Behavior theory and conditioning. New Haven: Yale University Press, 1956.

(Received for publication November 6, 1972.)

Amsel, A. The role of frustrative nonreward in noncontinuous 